\title{
Ticking all the boxes
}

\section{Nature Materials is extending editorial policies regarding transparency of reported data in manuscripts from the physical and life sciences.}

The reproducibility of scientific findings reported in peer-reviewed journals has lately been subjected to close scrutiny from the research community ${ }^{1}$. Several editors across the Nature family have engaged closely with researchers in various disciplines of the physical and life sciences, and identified the best-practice rules and standard requirements to accurately report scientific results. As a result of this collaboration, a few checklists summarizing these key reporting points have been adopted in our journals to help our authors present their findings according to the standards expected from their communities.

A checklist for life sciences manuscripts was introduced in 2013 in Nature ${ }^{2}$ and Nature journals ${ }^{3}$. So far, we have been asking authors of manuscripts sent out for external peer review to fill in the editorial policy checklist $^{4}$, which is then made available to the reviewers. Since June 2017 we are also requesting that a life sciences reporting summary ${ }^{5}$ is completed, which will be subsequently published with all accepted papers. These documents are intended to aid the reproducibility of life sciences research results by improving transparency in experimental design, data analysis, exclusion, replication, randomization and blinding. Some of the important aspects of this life sciences checklist are highlighted below. We encourage the inclusion of information on availability of raw data and materials, and require details about the validity of antibodies as well as authenticity of cell lines. Authors reporting experiments containing gels and blotting membrane data are encouraged to provide positive and negative controls in addition to molecular size markers on each gel and blot. It is mandatory for studies reporting novel sequences and structures of proteins, DNA, RNA and other biomolecules, and containing microarray data to deposit their datasets into community-endorsed, public repositories, providing accession codes in the data availability statement ${ }^{6}$. Studies involving the use of human embryonic stem cells are encouraged to follow the guidelines from the International Society for Stem Cell Research ${ }^{7}$. Animal studies are encouraged to follow the ARRIVE guidelines ${ }^{8}$, and a statement on ethics committee approval has to be included in the Methods section. For studies involving human subjects, a confirmation that informed consent was obtained from all subjects will be required in addition to the ethics committee approval statement. Studies reporting phase II and III clinical trials are required to conform to the CONSORT statement (http://www.consortstatement.org). There are also accessory reporting summaries for studies involving MRI, flow cytometry and ChIP-seq that must be completed.

Similar concerns on transparency and reproducibility have also been raised in

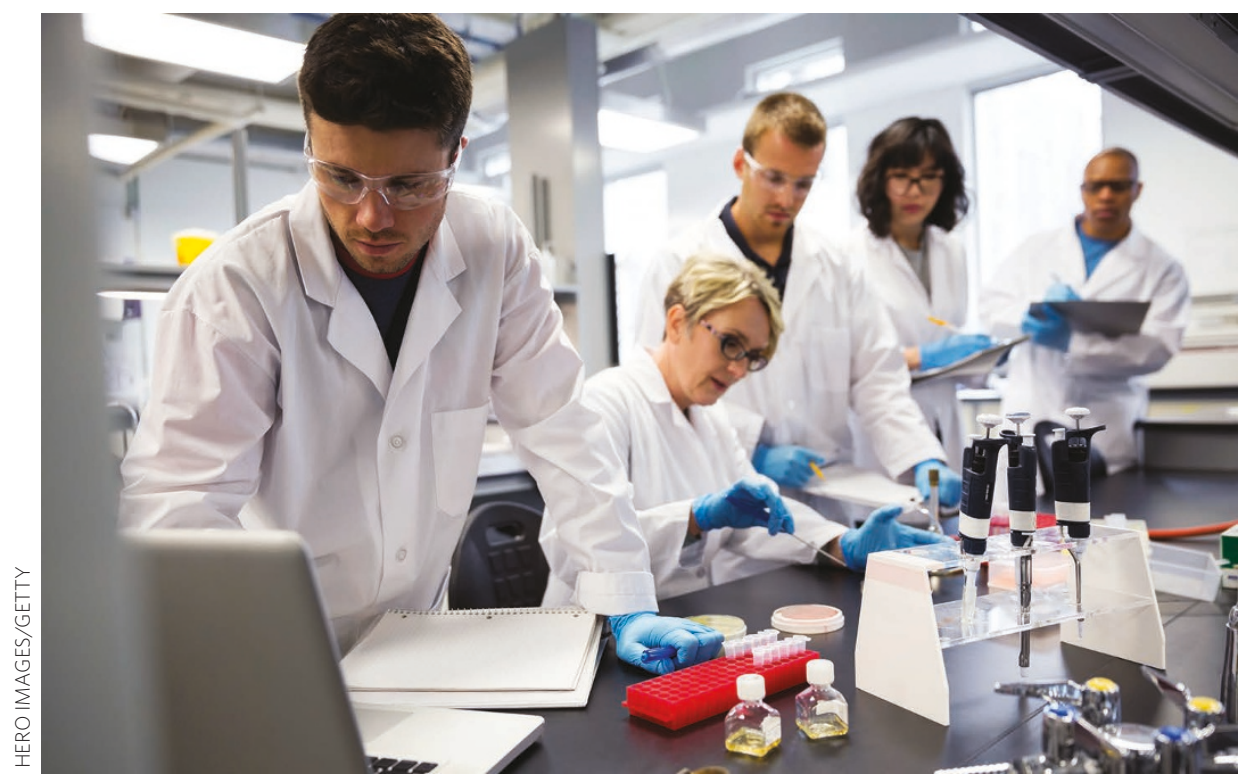

some physical sciences communities. Almost two years ago, Nature Materials and other Nature journals introduced a checklist for papers reporting solar cells performance; the aim was to ensure that information essential to describe the characterization techniques and properties of photovoltaic cells (including the voltage scan conditions, the use of masks during testing and statistical information about the reproducibility of the performance) was clearly included in the relevant papers. The initiative has now become a standard, with the checklist having been recently revised according to the suggestions received in the past few months from the solar cell community.

Reproducibility issues have also reached the laser community. Lasers come in so many different flavours at the moment, depending on the material, the output power and applications. Given the plethora of papers we receive with claims of lasing, ensuring that crucial information (such as a clear threshold, linewidth narrowing and number of devices tested) is clearly stated feels like a natural step. We are currently asking the authors of these manuscripts to fill in a checklist ${ }^{10}$ on submission, which is then shared with the reviewers. Several other Nature journals have also introduced similar guidelines ${ }^{11}$.

In all the cases mentioned above, the input from prominent researchers was vital, not only to support the editorial initiatives but also to give helpful feedback on the points to be included. We kindly thank them and the broader communities for embracing the checklists we have introduced; we are certain that transparency issues across the research landscape can only be successfully addressed when all sides whether researchers, publishers or funding agencies - play their part.

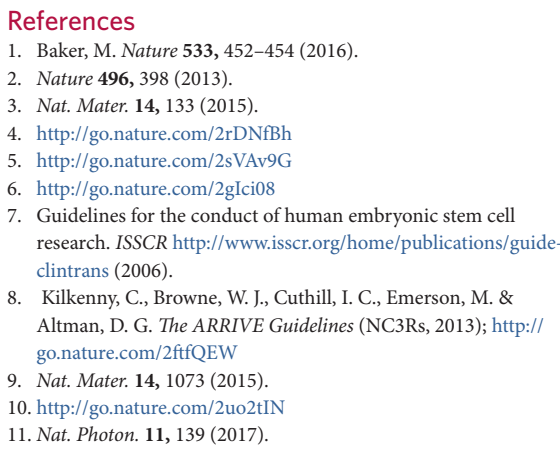

